\title{
Managing a Renal Transplant Programme During the COVID-19 Pandemic: Practical Experience from a Singapore Transplant Centre
}

Terence Kee, ${ }^{4,9}{ }_{M B B S,}$ FAMS, FRCP, Valerie $\underline{\text { Gan HL}, ~}{ }^{5,8}{ }_{M B B S,}$ MRCS, FAMS(Urology), Jasmine Chung Shimin, ${ }^{2,7}{ }_{M B B S,}$ MRCP,

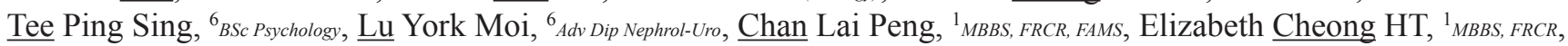

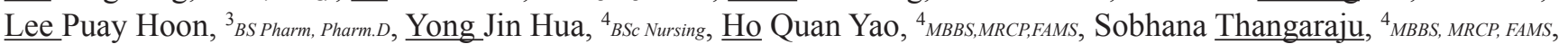

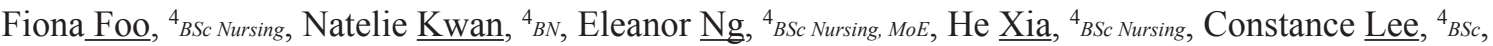
Shannon Boey, ${ }^{7}$ BSC Nursing, Marjorie Foo, ${ }^{4}$ MRCP, FRCP (Glas), FAMS, Tan Chieh Suai, ${ }^{4}$ MBBS, MRCP, FAMS

\begin{abstract}
Introduction: Coronavirus Disease 2019 (COVID-19) has significantly affected the way healthcare is delivered in Singapore. Healthcare services such as renal transplantation had to rapidly adjust and meet the needs to (1) protect patients and staff, (2) ramp up, conserve or redeploy resources while (3) ensuring that critical services remained operational. This paper aims to describe the experience of the renal transplant programme at the Singapore General Hospital (SGH) in responding to the risks and constraints posed by the pandemic. Methods and Materials: This is a review and summary of the SGH renal transplant programme's policy and protocols that were either modified or developed in response to the COVID-19 Pandemic. Results: A multi-pronged approach was adopted to respond to the challenges of COVID-19. These included ensuring business continuity by splitting the transplant team into different locations, adopting video and tele-consults to minimise potential patient exposure to COVID-19, streamlining work processes using electronic forms, ensuring safe paths for patients who needed to come to hospital, ring-fencing and testing new inpatients at risk for COVID-19, enhancing precautionary measures for transplant surgery, ensuring a stable supply chain of immunosuppression, and sustaining patient and staff education programmes via video conferencing. Conclusions: Though the COVID-19 pandemic has reduced access to kidney transplantation, opportunities arose to adopt telemedicine into mainstream transplant practice as well as use electronic platforms to streamline work processes. Screening protocols were established to ensure that transplantation could be performed safely, while webinars reached out to empower patients to take precautions against COVID-19.
\end{abstract}

Ann Acad Med Singap. 2020;49:652-60

Key words: COVID-19, Nephrology, Public Health, Renal Transplant, Transplantation, Urology

\section{Introduction}

The COVID-19 (Coronavirus Disease 2019) pandemic caused by Severe Acute Respiratory Syndrome Coronavirus 2 (SARS-CoV-2) is a global pandemic of epic proportion with far-reaching consequences. Apart from the unprecedented impact on public health, never before has the world seen so many countries locking down at various levels that has shell-shocked economies and plunged the world into its deepest recession since the Second World War.
In anticipation of COVID-19 reaching its shores, Singapore set up a Multi-Ministry Task Force on COVID-19 on 22 January 2020, while the pandemic alert system (termed 'Disease Outbreak Response System Condition' (DORSCON) in Singapore) was raised from green to yellow (Table 1). ${ }^{1,2}$ With uncanny timing, the first case of COVID-19 in Singapore was reported in a tourist from Wuhan on 23 January 2020. As the number of COVID-19 cases increased with reports of local transmission, the taskforce quickly

\footnotetext{
${ }^{1}$ Department of Diagnostic Radiology, Singapore General Hospital, Singapore

${ }^{2}$ Department of Infectious Diseases, Singapore General Hospital, Singapore

${ }^{3}$ Department of Pharmacy, Singapore General Hospital, Singapore

${ }^{4}$ Department of Renal Medicine, Singapore General Hospital, Singapore

${ }^{5}$ Department of Urology, Singapore General Hospital, Singapore

${ }^{6}$ Renal Transplant Programme, Singapore General Hospital, Singapore

${ }^{7}$ SingHealth Duke-NUS Transplant Centre, Singapore

${ }^{8}$ Kidney and Pancreas Transplantation, SingHealth Duke-NUS Transplant Centre, Singapore

${ }^{9}$ Renal Transplantation, SingHealth Duke-NUS Transplant Centre, Singapore

Address for Correspondence: Dr Terence Kee, Renal Medicine Office, Level 3, 20 College Road, Academia Building, Singapore 169586

Email: terence.kee.y.s@singhealth.com.sg
} 
raised the DORSCON further from yellow to orange in February 2020 (Table 1). A wide range of public health measures was also implemented and these included (1) heightened vigilance, screening and containment of suspected COVID-19 cases, (2) ramping up testing capabilities, (3) aggressive contact tracing, (4) reducing non-essential healthcare services to redeploy manpower and resources for COVID-19, (5) expansion of negativepressure isolation wards and intensive care units in the public sector hospitals, and (6) stockpiling of personal protective and other medical equipment. ${ }^{3}$

Since January 2020, Singapore's first wave of COVID-19 infection (Table 2) has presented itself in 4 phases, reflecting the evolving epidemiology of COVID-19 in the country. ${ }^{4}$ In response to the escalating COVID-19 situation, a set of preventive measures was implemented to halt the rising incidence of community cases between 7 April 2020 and 1 June 2020; this was termed the "circuit breaker". These measures included movement restrictions, closure of all schools, dine-in services at food and beverage outlets, retail stores and most workplaces except those in key economic sectors or providing essential services.

As infrastructure and policies were being put in place to combat COVID-19, we recognised that specialty-specific policies and protocols had to be drawn up as well. Similarly, the Renal Medicine Unit at the Singapore General Hospital (SGH), an academic medical centre, acted quickly to modify our services to (1) protect patients and staff, (2) ramp up, conserve or redeploy resources while (3) ensuring that critical services remained operational. ${ }^{5,6}$ This paper aims to share the Renal Transplant Unit's experience of adapting to COVID-19 precautions, and countermeasures from the perspective of its renal transplant programme.

\section{Reorganisation and Enhancing Mobility of the Workforce}

The renal transplant programme at SGH is organised into 4 teams covering core services such as wait-list management, living kidney donor transplantation, posttransplantation follow-up, and administrative/registry support. When the DOSRCON alert was raised from yellow to orange on 7 February 2020 (Table 2), the staff split into 2 teams to be stationed at 2 different buildings of the hospital campus (SGH and the Academia building, which houses offices and laboratories). Each team consisted of staff from the 4 core services of the programme, and hence was able to work as a functional unit on its own. This is part of our business contingency plan to ensure that the programme would continue to function should any team be quarantined because of
COVID-19. Telecommuting via Zoom, a cloud-based video conferencing tool, replaced physical meetings. In addition, strict social distancing measures were put in place while physical interaction between the 2 teams was prohibited. Working hours were also adjusted so that staff could avoid the rush hour commuter traffic, while a roster was set up to schedule staff to take turns to work from home.

Staff were mandated to wear masks at work, have their meals separately from each other and asked not to engage in social activities with each other after office hours. Staff were also initially forbidden to travel or take leave unless for exceptional indications, but this was later loosened to allow for some leave (but not overseas travel) each month as the number of community cases stabilised. In fact, as the prospects of the COVID-19 pandemic resolving soon became less realistic, staff were encouraged to take short leave during each month to rest and recover.

\section{Telemedicine in the Age of COVID-19}

Soon after the first few imported cases of COVID-19 were diagnosed in late January 2020, SGH instructed clinical departments to reduce outpatient clinic workload by $50 \%$ to reduce patient visits to the hospital as well as to prepare resources for an incoming wave of COVID-19 infections. As a result, physicians reviewed the electronic records of transplant recipients and living kidney donors to determine which patients could safely defer their appointments. However, as the COVID-19 outbreak escalated in Singapore over the next few weeks, it was clear that appointments could not be postponed for prolonged periods of time. As a result, applications for approval to start video consult services were put up. Video consult services eventually started in May 2020, following regulatory approvals and completion of staff training on video consultation. While the programme was waiting for video consultation services to be set up, the number of physical clinic visits was minimised by physicians performing phone consults with patients who were deemed stable enough not to require a hospital visit.

Transplant counselling services by transplant coordinators also shifted to video counselling using Zoom with surprisingly positive benefits. It was easier to engage and counsel potential transplant candidates and their families because the counselling could be done in the convenience of their home or workplace. Furthermore, more family members were able to join in the video counselling, which meant that a larger pool of potential living kidney donors could be reached. Video counselling also circumvented the movement restriction 


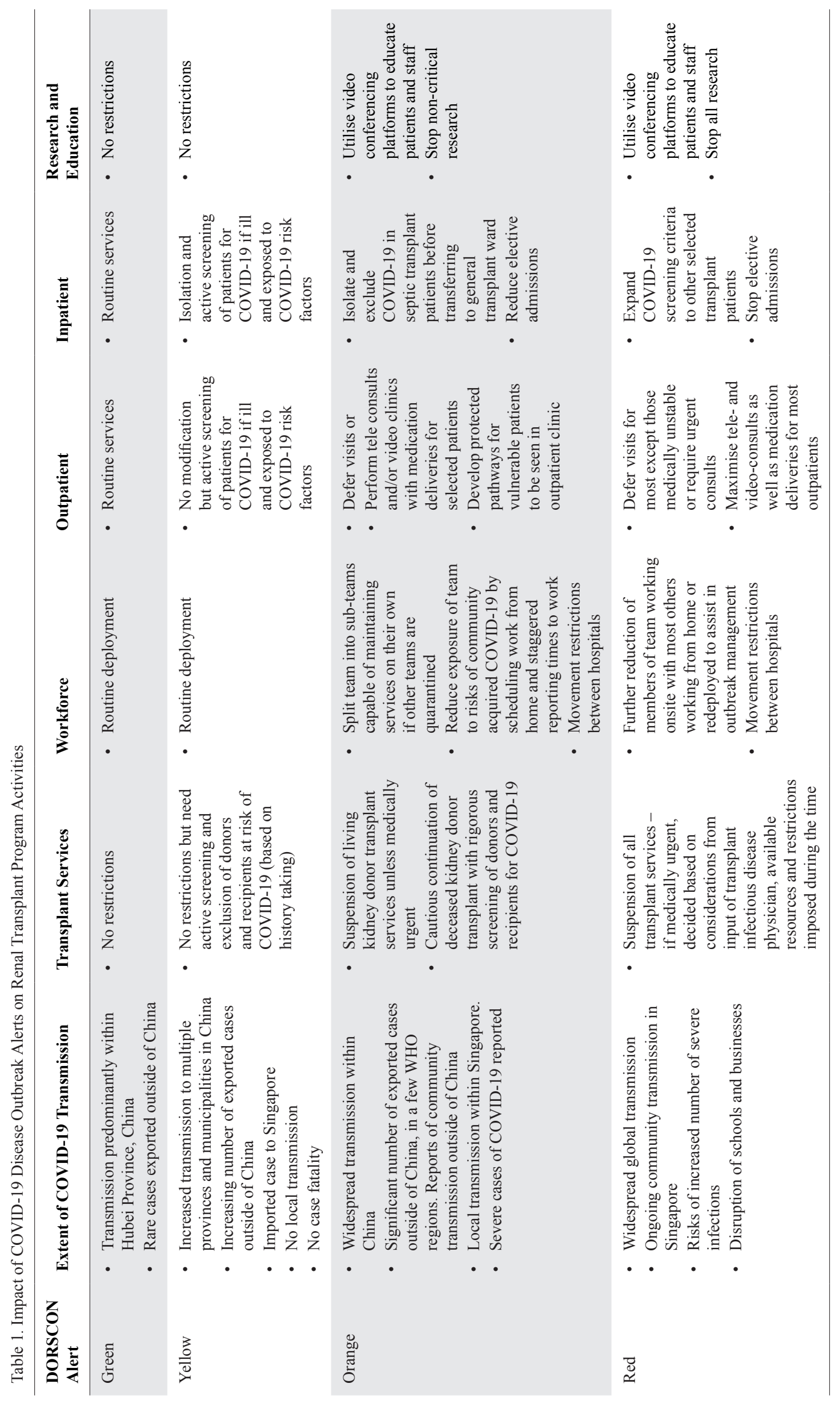


on transplant coordinators performing transplant counselling at the regional hospitals.

The COVID-19 pandemic also provided an opportunity for the transplant coordinators to review and streamline their counselling work processes further. So, instead of manually going through screening checklists with the patients and their potential donors, transplant coordinators created digital forms using FormSG, a form builder tool developed by Singapore's Government Technology Agency. As a result, the electronic screening checklist forms could be emailed to patients for them to fill up and return in a secured manner.

Transplant pharmacists are an integral part of the patient care workflow at the transplant centre. Prior to COVID-19, all patients seen at the transplant centre would be reviewed by the transplant pharmacist. However, as the number of video and tele-consults increased, transplant pharmacists also had to adapt to counselling through phone or video calls. There was also ramping up of medication service delivery to reduce crowds in the dispensing pharmacy, and physicians were instructed to prescribe additional 2 weeks of immunosuppressants to their patients' supply in case of quarantine.

\section{Reconfiguration of Patient Flow at the Outpatient Transplant Centre}

The programme also reconfigured the outpatient transplant centre so that patients who were heavily immunosuppressed, such as recent transplants or those who had received treatment of rejection, could enter the transplant centre by the rear entrance and perform their consult with the physician in a dedicated room away from the main entrance and waiting area. Patients were given staggered appointments so that there would be no waiting in the rear area, and different healthcare providers, e.g. physician, pharmacist and transplant coordinator, would take turns to counsel the patient in the same room.

The transplant centre also had dedicated areas for patients waiting for consults and those waiting for blood drawing, ensuring social distancing measures were enforced. Transplant coordinators were tasked to periodically assess the situation at the transplant centre, monitoring waiting times and ensuring that there was no overcrowding. Outpatient swabbing capability was also created to test outpatients for COVID-19.

\section{Modification of Inpatient Services}

The need to segregate patients who are COVID-19 suspects stems from our experience in 2003, where there were nosocomial transmissions of SARS from infected patients who were nursed in the open wards. ${ }^{7}$ In response, at the start of the current COVID-19 pandemic, our general wards were reconfigured to become respiratory surveillance wards (RSW) where all patients with respiratory tract infections (including kidney transplant recipients) could be screened. ${ }^{8}$ If COVID-19 was excluded, the patient could be transferred to the general ward for continuation of care. Conversely, if they were positive, they would be transferred to the COVID-19 designated isolation ward. As the COVID-19 pandemic progressed, it became clear that COVID-19 was not purely a respiratory tract infection and the admission criteria into the RSW broadened to include any patient with fever or other risk factors for COVID-19. Hence, kidney transplant recipients with diarrhoea were also considered COVID-19 suspects and underwent testing for SARS$\mathrm{CoV}-2$. This was because there were reports that kidney transplant recipients with COVID-19 could present primarily with gastroenteritis. ${ }^{9}$ The RSW proved useful to mitigate possible patient-healthcare worker transmission of COVID-19, as $1.61 \%$ of the 1,178 patients from the RSW tested positive for SARS-CoV-2, none of whom were kidney transplant recipients. ${ }^{8}$ It is interesting to observe that during the period of January to August 2020, only 28 kidney transplant recipients were admitted to the RSW at SGH. Among these 28 patients, 18 were diagnosed with viral respiratory tract infections (RTI) which was markedly lower than the 30 kidney transplant recipients diagnosed with viral RTI during the same

Table 2. Phases of COVID-19 Infections in Singapore

\begin{tabular}{lll}
\hline Phase & Period & People affected \\
\hline 1 & 23 January to 1 February 2020 & Chinese tourists and Singapore residents coming to Singapore from Wuhan \\
2 & 3 February to 10 March 2020 & Singapore residents linked to infected Chinese visitors \\
3 & 11 March to 1 April 2020 & $\begin{array}{l}\text { Largely Singapore residents returning from countries which has locked down due } \\
\text { to COVID-19 }\end{array}$ \\
\hline 4 & 5 April to present & Foreign workers largely from dormitories \\
\hline
\end{tabular}


period in 2019. This suggests that patients were taking precautionary measures seriously, thus avoiding respiratory tract infections from the community or workplace.

Physicians from the transplant team also took turns to cover RSW while others in the team continued to care for kidney transplant recipients in other parts of SGH. During the initial outbreak period of February to March 2020, the transplant team first divided themselves into 2 teams that strictly covered different areas of SGH and avoided contact with one another. However, it was soon realised that PPE was an effective defence against COVID-19 as no healthcare provider was getting infected by COVID-19 while at work. ${ }^{10}$ As a result, strict segregation was discontinued, and cross-cover to different patient areas was permitted. Nevertheless, members of the teams continued to minimise unnecessary in-person meetings and adopted telecommuting platforms to discuss cases.

\section{Enhancing Screening for COVID-19 in Kidney Transplant Procedures}

As the first reports of community transmission of COVID-19 began streaming in from 4 February 2020 onwards, it was decided to slow down the processing of referrals for transplantation in anticipation that such activities would soon be suspended just as it was during the SARS outbreak in 2003. As a result, the transplant programme performed only 2 living kidney donor transplants on 20 January 2020 and 3 February 2020, just prior to the start of community spread of COVID-19. By 19 February 2020, the Ministry of Health issued an advisory that living kidney donor transplantation services could continue but deceased donor and tissue transplantation services were to be suspended unless it was medically urgent. Thus, the last living kidney donor transplant (spousal) was performed on 18 March 2020, but with special precautionary measures put in place. These included comprehensive counselling of the donor-recipient pair and their care givers (parents) on minimising contact with the community, frequent hand hygiene, and proper masking in the 14 days prior to the admission for transplantation. ${ }^{11}$ The donor and recipient also underwent polymerase chain reaction (PCR) testing for SARS-CoV-2 one week and one day before surgery. They were tested negative for COVID-19 and the transplant surgery was uneventful. Both patient and donor remained stable without COVID-19 infection as of 31 August 2020.

As local transmissions of COVID-19 increased at the end of March 2020, the living kidney donor programme was suspended at our hospital due to safety considerations.
When Singapore went into "circuit breaker" mode in early April 2020, the Ministry of Health issued a second advisory to suspend living kidney donor transplants unless it was medically urgent; deceased donor kidney transplants were permitted. Stringent screening measures were put in place to exclude COVID-19 in deceased donors. These included obtaining a detailed history to screen for COVID-19 risk factors, testing serial respiratory specimens for SARS-CoV-2 and performing computed tomography (CT) of the chest. ${ }^{12}$ The recommendation for CT chest as part of the deceased donor workup was based on previous reports that COVID-19 pneumonia could be detected on CT even prior to the detection of SARS-CoV-2 on PCR assays. ${ }^{13-14}$ However. as the number of community cases of COVID-19 decreased over time, CT became optional as emerging evidence suggested that the positive predictive value of CT to diagnose COVID-19 was low in areas of low COVID-19 prevalence. ${ }^{15}$

At present, the deceased donor kidney transplant programme remains open. Should an organ donor offer be made, the medical staff would first contact the potential recipient(s) and obtain a detailed history to assess for COVID-19 exposure/symptoms. The patient(s) with no identifiable risk factors for COVID-19 would then be asked to come to hospital. Each of them would be admitted to a single room, and screened for COVID-19; respiratory specimens for SARS-CoV-2 PCR would be collected and chest radiology performed. ${ }^{12}$ Nursing and medical staff would be in full PPE (i.e. N95, face shield or goggle, gown and gloves) while nursing the patient until COVID-19 has been excluded.

It was through this protocol that we picked up presymptomatic COVID-19 in one of our potential recipients. She had no identifiable risk factors and was asymptomatic at the time of recall for transplant surgery. ${ }^{16}$ When she was tested positive for COVID-19, she was bypassed for transplant and transferred to the COVID-19 designated isolation ward. The patient developed a fever the next day but subsequently recovered fully. Fortunately, there were no cases of nosocomial transmission from this index case owing to the use of full PPE.

With the lifting of the circuit breaker on 1 June 2020, the Ministry of Health issued a 3 advisory to permit living kidney donor transplantation on 3 July 2020 as permitted by the prevailing available resources and the COVID-19 situation. As a result, the first living kidney donor transplantation was performed on 9 September 2020 with the previously described precautionary measures being taken. 


\section{Performing Surgery Safely}

The SGH Urology Operating Room (OR) is a small, standalone complex away from the main OR complex, and comprises 3 separate ORs. This is where our living kidney donor transplants are performed, with the donor and recipient in adjacent ORs. Deceased kidney donor transplantations performed after office hours are performed in the main OR complex.

During this outbreak, the Urology OR complex was designated for surgery in patients suspected or confirmed to have COVID-19. As such, our elective living kidney donor transplants had to be performed in the main OR complex. We were fortunate to have been able to adhere to our usual practice of using 2 adjacent ORs (this minimises the ischemia time as the recipient surgery is commenced in the other OR when the donor surgeon is preparing to clamp the renal vessels). The main ORs were designed to be at positive pressure relative to the surrounding air, with a high frequency of air changes ( 25 per hour).

The transplant surgical team was minimised to 3 surgeons, all of whom did not care for COVID-19 patients or front any high-risk areas. As per hospital protocol, all aerosol-generating procedures required staff to be in full PPE including N95 respirators or powered air-purifying respirators (PAPR) (if they failed N95 mask-fitting), goggles/face shields, gowns and gloves. This was the practice for all surgical intubations/ extubations, and thus the scrub and surgical teams were not allowed into the OR till intubation was performed. It was not necessary for the surgeons to don 95 masks as the transplant donors/recipients had been screened thoroughly and deemed unlikely to have COVID-19, thus allowing surgery to proceed.

\section{Ensuring Supply Chain}

When DOSRCON level was raised from yellow to orange, the renal transplant programme engaged pharmaceutical companies and received assurance that the import of immunosuppressive drugs would not be disrupted arising from lockdowns in countries affected by COVID-19. The drug supply chain for all drugs was also closely monitored to ensure there was no disruption in therapy. In order to conserve precious resources like plasma exchange kits, intravenous immunoglobulin, intensive care beds and antibiotics, the programme avoided performing high-immunological risks kidney transplants using expanded criteria deceased donors and $\mathrm{ABO} / \mathrm{HLA}$ incompatible living donor kidney transplant. Renal physicians were also advised not to reduce immunosuppression without a strong indication so as to avoid triggering antibody mediated rejections in the existing transplant population. Annual screening tests like ultrasound and $\mathrm{CT}$ imaging were also deferred to conserve radiological resources for COVID-19.

\section{Impact on Transplant Workload and Outcomes}

The COVID-19 has had a major impact on transplant workload. During the period from January to August 2020, kidney transplant rates had been more than halved, with only 6 kidney transplants performed compared to 14 during the same period in 2019. However, despite the reduction in clinical exposure and redeployments, clinical outcomes remained good with no patients experiencing rejection, and no deaths or graft loss. More importantly, none of these patients were infected with COVID-19 after transplantation despite being heavily immunosuppressed.

Workload across other areas of the transplant service was also reduced, especially with respect to referrals for kidney transplantation (Table 3). However, despite a lack of timely access to deceased donor kidney transplantation, the mortality rate of patients on the waiting list for such transplantation during the period from January to May did not increase (1 death each in 2019 and 2020). Notably, outpatient follow-up clinics were not significantly compromised, likely because video clinics replaced physical clinics. This may have maintained the quality of care as the number of inpatient transplant admissions did not increase during the COVID-19 pandemic.

Despite this reduction of workload, transplant staff were kept busy through deployment to COVID-19 related units e.g. perimeter screening, fever triage area, RSW and Forward Assurance and Support Teams (FAST) at the foreign worker dormitories. Transplant staff also had to organise live webinars to educate patients about precautions against COVID-19, and send out surveys to examine the attitudes and emotional responses to getting transplanted or being a transplant recipient during the COVID-19 pandemic. These survey questionnaires were sent out to determine what areas of concerns needed to be addressed by the programme so that appropriate support services could be given. Notably, preliminary analyses of these surveys suggest that over $80 \%$ of survey respondents felt they were unlikely to contract COVID-19 and disagreed that the quality of healthcare during the pandemic had deteriorated. Furthermore, only $14.3 \%$ of respondents had some level of emotional disturbance on the Depression, Anxiety, Stress Scale (DASS). This is certainly a testimony of the robust nation-wide response and communication efforts with regard to COVID-19. 
Table 3. Impact of COVID-19 on Kidney Transplant Services at the Singapore General Hospital

\begin{tabular}{lcc}
\hline & January to May 2019 & January to May 2020 \\
\hline Living kidney donor transplant & 11 & 3 \\
\hline Deceased kidney donor transplant & 3 & 0 \\
\hline Referrals for wait-list & 63 & 18 \\
\hline Referrals for living kidney donor transplantation & 63 & 42 \\
\hline Transplant assessment clinics & 117 & 61 \\
\hline Outpatient transplant follow-up clinics & 211 & 202 \\
\hline Inpatient transplant admissions & 314 & 270 \\
\hline
\end{tabular}

\section{Impact on Transplant Research and Education}

Owing to movement restrictions and social distancing, all educational activities were conducted on the Zoom platform, while research involving collection of blood samples was halted. Once again, we appreciate the value of Zoom to hold video classes as it reduced the travel time required to attend physical classes, and hence improved both student and patient attendance rates. This was especially useful for our programme as we provide transplant training to senior residents posted to several hospitals such as Changi General Hospital, Sengkang General Hospital and Tan Tock Seng Hospital. As a result, we will continue this mode of tele-education even after the COVID-19 pandemic has subsided.

\section{Learning in a Time of Crisis}

The COVID-19 pandemic has significantly affected the access of patients with end-stage kidney failure to kidney transplantation, a treatment which significantly improves a patient's survival and quality of life. However, it has also revealed alternative ways to deliver transplant services and uncover opportunities to build up pandemicready capabilities.

One of the greatest impacts the pandemic has was the accelerated adoption of telemedicine in Singapore. We were abruptly forced to adopt telemedicine quickly and in all facets of transplant clinical practice. It sparked a culture shift for staff to be trained and be well versed in telemedicine. We also realised that infrastructure was lagging behind the need to adopt telemedicine into mainstream clinical practice, as not all areas of the hospital was able to offer strong WiFi signals. Finally, in order for telemedicine to be applied safely and effectively, there is an urgent need to educate and encourage patients to adapt to this new modality of clinical consult while assuring them that care will not be compromised. In our experience, $35 \%$ of patients invited to participate in video clinics did not do so as they were not familiar with the technologies required or the telecommuting platform despite video and written instructions given to them by the transplant coordinators. Another $10 \%$ had to convert to phone consults because of poor WiFi connectivity. Nevertheless, we have appreciated the value of telemedicine and would continue its practice even when the COVID-19 pandemic is no more. Importantly, it appears safe as only $2 \%$ of patients participating in video clinics experienced a hospital admission within 1 month of the video consult. This would be especially helpful in reducing the number of physical visits of very stable kidney transplant recipients and increase the accessibility of patients and their loved ones to kidney transplant education by transplant coordinators. There are also hidden patient-centric advantages such as reduction in transportation expenditure and the ability to remain at the workplace during a video consult.

Social distancing has become the new normal of living during the COVID-19 pandemic. In our efforts to recreate smaller teams deployed to different physical areas to work, we learnt that digital mobility of staff was hampered by problems such as (1) limited office space and desktop connection points, which reduced the flexibility of teams to split and be redeployed in different areas; and (2) not all staff could work from home owing to the lack of WiFi connection and data secured laptops. Fortunately, we were able to circumvent those problems, but the pandemic does highlight the importance of improving our digital platforms for the transplant services through the greater use of secure mobile electronic devices (including 
laptops) and empowering staff with access to good WiFi connections at home (e.g. portable WiFi).

We also realised that the existing infrastructure in our outpatient facilities has not been designed and built with social distancing in mind. Overcrowding during peak hours of blood draw and the lack of dedicated space for highly vulnerable transplant recipients were concerns that we had to quickly develop solutions within the constraints of existing infrastructure. Moving forward, it would be critical for us to relook at our outpatient workflow and processes as well as reconfiguring infrastructure to facilitate social distancing.

The pandemic has demonstrated the enormous impact of wearing a facemask in reducing respiratory tract infections in kidney transplant recipients. With Singapore's introduction of mandatory masking up in public on 14 April 2020, we did not see any patients admitted with viral respiratory tract infections from June 2020 onwards. We believe that even after COVID-19 pandemic has ended, masking up should be strongly encouraged for our kidney transplant recipients, especially when they visit crowded places. This may not be too difficult to do now that wearing of masks has become a socially acceptable thing to do.

Finally, we were fortunate that the supply chain of brand-name immunosuppressive drugs was not disrupted by the COVID-19 pandemic. Nevertheless, it does highlight a potential vulnerability for future andemics, and our transplant pharmacists are exploring the possibility of procuring generic immunosuppressive drugs as an emergency back-up.

\section{Conclusion}

John F. Kennedy, the 35th President of the United States of America, was quoted to have said, "When written in Chinese, the word crisis is composed of two characters - one represents danger, and the other represents opportunity." This holds true for the COVID-19 pandemic, which has disrupted kidney transplant services but also challenged the transplant programme to review and improve on its processes with gains. With the progressive reopening of our economy and borders with other countries, it is critical for us to continue to build on the adaptations arising from the pandemic, especially when an effective vaccine remains unavailable. We also believe that the transplant programme has done well as a benefactor of nationwide efforts to ensure that the numbers of COVID-19 infected cases did not overwhelm the healthcare system in Singapore. Unlike other programmes, we have
COVID-19 testing capabilities (compared to $47 \%$ of transplant centres in the United States) and are fortunate not to experience the high numbers and mortality of COVID-19 infected kidney transplant recipients in other countries (Table 4). ${ }^{17-26}$ As with many crises, the COVID-19 pandemic has also brought out the courage, resilience and tenacity of our staff as well as demonstrated the remarkable abilities for different departments at SGH to quickly and effectively work together on urgent solutions. There is no doubt that we will emerge from the COVID-19 pandemic stronger and better than before.

\section{REFERENCES}

1. Lin RJ, Lee TH, Lye DC. From SARS to COVID-19: the Singapore journey. Med J Aust. 2020 Jun; 212(11):497-502.

2. Chung SJ, Tan EK, Kee T, et al. Practical considerations for solid organ transplantation during the COVID-19 global outbreak: the experience from Singapore. Transplant Direct 2020 May 8;6(6): e554. doi: 10.1097/TXD.0000000000001002.

3. Wong J, Leo YS, Tan CC. COVID-19 in Singapore - Current Experience. Critical global issues that require attention and action. JAMA 2020 Apr 7;323(13):1243-1244.

4. Update on COVID-19 (Coronavirus Disease 2019) Local Situation. https://www.moh.gov.sg/covid-19. Accessed on 23 June 2020.

5. En Khoo BZ, See YP, Kam Koh TJ, Yeo SC. Coronavirus Disease 2019 (COVID-19) and Dialysis: The Experience in Singapore. [published online ahead of print, 2020 May 13]. Kidney Med. 2020 May 13;2(4)381-384. doi: 10.1016/j.xkme.2020.05.002.

6. Chua HR, Laren GM, Choong LH, Chionh CY, Khoo BZE, Yeo SC, et al. Ensuring Sustainability of Continuous Kidney Replacement Therapy in the Face of Extraordinary Demand: Lessons From the COVID-19 Pandemic. [published online ahead of print, 2020 Jun 4]. Am J Kidney Dis. 2020 Sep;76(3):392-400. doi: 10.1053/j. ajkd.2020.05.008.

7. Ong YY. SARS - Lessons from two Singapore General Hospital cases. Singapore Med J 2003 Aug;44(8): 420-2.

8. Wee LE, Hsieh JYC, Phua GC, Tan Y, Conceicao EP, Wijaya L, et al. Respiratory surveillance wards as a strategy to reduce nosocomial transmission of COVID-19 through early detection: The experience of a tertiary-care hospital in Singapore. (published online ahead of print, 8 May 2020). Infect Control Hosp Epidemiol. 2020 May 8; 1-6. doi: 10.1017/ice.2020.207.

9. Guillen E, Pineiro GJ, Revuelta I, Rodriguez D, Bodro M, Moreno A, et al. Case report of COVID-19 in a kidney transplant recipient: Does immunosuppression alter the clinical presentation? (published online ahead of print, 20 March 2020) Am J Transplant. 2020 Jul;20(7):18751878. doi: 10.1111/ajt.15874

10. Ng K, Poon BH, Kiat Puar TH, et al. COVID-19 and the Risk to Health Care Workers: A Case Report. Ann Intern Med. 2020 Jun 2;172(11):766767. doi:10.7326/L20-0175

11. Ho QY, Chung SJ, Gan VHL, Ng LG, Tan BH, Kee TYS. Highimmunological risk living donor renal transplant during the COVID-19 outbreak: uncertainties and ethical dilemmas. [published online ahead of print, 2020 Apr 26]. Am J Transplant 2020 Jul;20(7):1949-1951. doi: 10.1111/ajt.15949.

12. Ai T, Yang Z, Hou H, Zhan C, Chen C, Lv W, et al. Correlation of Chest CT and RT-PCR Testing in Coronavirus Disease 2019 (COVID-19) 
in China: A Report of 1014 Cases. [published online ahead of print, 2020 Feb 26]. Radiology. 2020 Aug;296(2):E32-E40. doi: 10.1148/ radiol.2020200642.

13. Fang Y, Zhang H, Xie J, Lin M, Ying L, Pang P, et al. Sensitivity of Chest CT for COVID-19: Comparison to RT-PCR. [published online ahead of print, 2020 Feb 19]. Radiology. 2020 Aug;296(2): E115-E117. doi: 10.1148/radiol.2020200432.

14. Xie X, Zhong Z, Zhao W, Zheng C, Wang F, Liu J. Chest CT for Typical 2019-nCoV Pneumonia: Relationship to Negative RT-PCR Testing. [published online ahead of print, 2020 Feb 12]. Radiology. 2020 Aug;296(2):E41-E45. doi: 10.1148/radiol.2020200343.

15. Parekh M, Donuru A, Balasubramanya R, et al. Review of the Chest CT Differential Diagnosis of Ground-Glass Opacities in the COVID Era. Radiology 2020 Jul 7; 202504. doi: 10.1148/radiol.2020202504.

16. Ho QY, Chung SJ, Low SCS, Chen R, Teh SP, Chan Y, et al. Evaluating potential deceased donor renal transplant recipients for asymptomatic COVID-19. Transplantation Direct:2020 May 22;6(6):e559. doi:10.1097/ TXD.0000000000001010.

17. Boyarsky BJ, Po-Yu Chiang T, Werbel WA, et al. Early impact of COVID-19 on transplant center practices and policies in the United States.Am J Transplant. 2020 Jul;20(7):1809-1818. doi:10.1111/ajt.15915

18. Fernandez-Ruiz M, Andres A, Loinaz C, et al. COVID-19 in solid organ transplant recipients: A single-center case series from Spain. Am J Transplant 2020 Jul; 20(7): 1849-1858. doi: 10.1111/ajt.15929.

19. Zhu L, Gong N, Liu B, et al. Coronavirus Disease 2019 Pneumonia in Immunosuppressed Renal Transplant Recipients: A Summary of 10 Confirmed Cases in Wuhan, China. Eur Urol 2020 Jun; 77(6): 748-754. doi: 10.1016/j.euro.2020.03.039.
20. Yi SG, Rogers AW, Saharia A, et al. Early Experiene With COVID-19 and Solid Organ Transplantation at a US High-volume Transplant Center. Transplantation 2020 Jun 1:10.1097/TP.000000000000339. doi: 10.1097/TP.0000000000003339. Online ahead of print.

21. Abrishami A, Samavat S, Behnam B, et al. Clinical Course, Imaging Features and Outcomes of COVID-19 in Kidney Transplant Recipients. Eur Urol 2020 Aug; 78(2): 281-286. doi: 10.1016/j. eururo.2020.04.064.

22. Hoek RAS, Manintveld OC, Betjes MGH, et al. COVID-19 in solid organ transplant recipients: a single-centre experience. Transplant Int 2020 May 27: 10.1111/tri.13662. doi.10.1111/tri.13662. Online ahead of print.

23. Alberici F, Delbarba E, Manenti C, et al. A single centre observational study of the clinical characteristics and short-term outcome of 20 kidney transplant patients admitted for SARS-CoV2 pneumonia. Kidney Int 2020 Jun; 97(6): 1083-1088. doi: 10.1016/j.kint.2020.04.002.

24. Devresse A, Belkhir L, Vo B, et al. COVID-19 Infection in Kidney Transplant Recipients: A Single-Centre Case Series of 22 Cases from Belgium. Kidney Med 2020 Jun 15; 2(4): 459-466. doi: 10.1016/j. xkme.2020.06.001.

25. Felldin M, Softeland JM, Magnusson J, et al. Initial Report from a Swedish High-Volume Transplant Centre After the First Wave of the COVID-19 Pandemic. Transplantation 2020 Aug 20. doi: 10.1097/ TP.0000000000003436. Online ahead of print.

26. Elias M, Pievani D, Randoux C, et al. COVID-19 Infection in Kidney Transplant Recipients: Disease Incidence and Clinical Outcomes. JAm Soc Nephrol 2020 Oct; 31(10):2413-2423. doi: 10.1681/ ASN.2020050639. Online ahead of print. 